\title{
Extensionality with respect to Indistinguishability Operators
}

\author{
D. Boixader J. Recasens \\ Sec. Matemàtiques i Informàtica \\ ETSAV, UPC \\ C. Pere Serra 1-15 \\ 08190 Sant Cugat del Vallès \\ Spain
}

\begin{abstract}
Extensionality is explored from different points of view. Extensional fuzzy subsets with respect to a fuzzy equivalence relation $E$ are considered as observable subsets with respect to the granularity generated by $E$. Interestingly, they are characterized as the fuzzy subsets that can be obtained as combinations of the fuzzy equivalence classes of $E$. Extensional mappings are characterized topologically and the set of extensional mappings between two universes is algebraically determined. As a result, an interpretation of type-2 fuzzy subsets as fuzzification of type-1 fuzzy subsets is provided.

Keywords: Extensional mapping, extensional fuzzy subset, indistinguishability operator, fuzzy equivalence relation, fuzzy topology, type-2 fuzzy subset.
\end{abstract}

\section{Introduction}

Extensionality, in its different aspects, is probably the most important property involved in the modelling of a fuzzy system in the presence of a fuzzy equality or equivalence relation. 
- Given a mapping $f: X \rightarrow Y$ and $E, F$ fuzzy equivalence relations (indistinguishability operators) on $X$ and $Y$ respectively, $f$ is $(E, F)$ extensional, or simply extensional, with respect to $E$ and $F$ when $E(x, y) \leq F(f(x), f(y))$ i.e., when the images of the objects $x$ and $y$ are more similar than the objects themselves. This is essential in fuzzy systems and in Approximate Reasoning, since it means that the consequences are closer than the antecedents [2] [7].

- A fuzzy set $\mu$ of $X$ is extensional with respect to an indistinguishability operator $E$ on $X$ when $T(E(x, y), \mu(x)) \leq \mu(y)$. Extensional fuzzy sets are the observable sets of $X$. Indeed, the last inequality fuzzifies the predicate

$$
\text { If } x \in \mu \text { and } x \sim y \text {, then } y \in \mu \text {. }
$$

In the crisp case, the extensional sets are combinations (unions) of equivalent classes of the equivalence relation $\sim$; that is, the sets of $X$ that can be observed with the granularity generated by the equivalence relation $\sim$.

The set $H_{E}$ of extensional fuzzy subsets of $X$ with respect to $E$ has in fact been identified with the set of fuzzy subsets of the quotient set $X / E\left(H_{E}=[0,1]^{X / E}\right)[4]$.

The fact that the extensional fuzzy subsets of $X$ can be obtained as combinations of the fuzzy equivalence classes of $E$ will be rigorously stated and proved in Proposition 3.8.

The set $H_{E}$ of extensional fuzzy subsets of $X$ with respect to $E$ has been extensively studied [8], As an important result, it has been proved that it is a fuzzy topology [8]. Also in [7] the sets that are the set of extensional fuzzy subsets of an indistinguishability operator $E$ have been algebraically characterized (Proposition 3.7). A deep look at this proposition allows of the characterization of the extensional fuzzy subsets of $X$ with respect to $E$ as the ones obtained as combination of the fuzzy equivalence classes of $E$ (Proposition 3.8) and provides a rigorous reason to naming observable the elements of $H_{E}$.

Extensionality of a fuzzy subset of $X$ with respect to $E$ is a special case of $(E, F)$-extensionality, since a fuzzy subset $\mu: X \rightarrow[0,1]$ of $X$ is extensional with respect to $E$ if and only if it is $(E, \overleftrightarrow{T})$-extensional, with $\overleftrightarrow{T}$ the 
biresiduation of $E$ (Proposition 2.4). This known result unifies and relates the results of extensional mappings and of extensional fuzzy subsets.

The biresiduation $\overleftrightarrow{T}$ plays an essential role in the study of extensionality and deserves special attention. This will be done at the end of Section 3 .

Section 4 studies the extensionality of mappings $f: X \rightarrow Y$. The first results relate extensionality with fuzzy continuity. Proposition 4.7 states that both concepts are equivalent in the presence of indistinguishability operators.

The sets of extensional mappings $f:(X, E) \rightarrow(Y, F)$ are characterized in Proposition 4.13 as a generalization of Proposition 3.7.

In Section 5 the relationship between extensionality and the generation of type-2 fuzzy sets is established.

\section{Preliminaries}

This section contains some definitions and properties related to $T$-indistinguishability operators that will be needed later. Throughout the paper $T$ will denote a left continuous t-norm.

Definition 2.1. Let $T$ be a t-norm,

- The residuation $\vec{T}$ of $T$ is defined by

$$
\vec{T}(x \mid y)=\sup \{\alpha \in[0,1] \mid T(x, \alpha) \leq y) .
$$

- The biresiduation $\overleftrightarrow{T}$ of $T$ is defined by

$$
\overleftrightarrow{T}(x, y)=T(\vec{T}(x \mid y), \vec{T}(y \mid x))
$$

\section{Example 2.2.}

- If $T$ is the Minimum t-norm, then

$$
\overleftrightarrow{T}= \begin{cases}\min (x, y) & \text { if } x \neq y \\ 1 & \text { otherwise }\end{cases}
$$

- If $T$ is a continuous Archimedean t-norm and $t$ an additive generator of $T$, then

$$
\overleftrightarrow{T}(x, y)=t^{[-1]}(|t(x)-t(y)|)
$$

In particular, 
- If $T$ is the t-norm of Lukasiewicz, then

$$
\overleftrightarrow{T}(x, y)=1-|x-y|
$$

- If $T$ is the Product t-norm, then

$$
\overleftrightarrow{T}(x, y)=\min \left(\frac{x}{y}, \frac{y}{x}\right)
$$

Definition 2.3. A fuzzy relation $E$ on a set $X$ is a T-indistinguishability operator on $X$ if and only for all $x, y, z$ of $X$ satisfies the following properties

- $E(x, x)=1$ (Reflexivity)

- $E(x, y)=E(y, x)$ (Symmetry)

- $T(E(x, y), E(y, z)) \leq E(x, z)$ (Transitivity)

$T$-indistinguishability operators extend the concept of equivalence relation and equality to the fuzzy framework and they are also called fuzzy equivalence and fuzzy equality relations. $E(x, y)$ can be viewed as the degree of equivalence, equality or indistinguishability between $x$ and $y$. A general panorama on $T$-indistinguishability operators can be found in [12].

The biresiduation $\overleftrightarrow{T}$ of a t-norm is a $T$-indistinguishability operator on $[0,1]$. That is why it is common to call it the natural $T$-indistinguishability operator associated to $T$ and denoted by $E_{T}$.

Proposition 2.4. Let $\mu$ be a fuzzy subset of $X$. The fuzzy relation $E_{\mu}$ on $X$ defined for all $x, y \in X$ by

$$
E_{\mu}(x, y)=E_{T}(\mu(x), \mu(y))
$$

is a T-indistinguishability operator.

Proposition 2.5. Let $\left(E_{i}\right)_{i \in I}$ be a family of T-indistinguishability operators on a set $X$. Then $\inf _{i \in I} E_{i}$ is a T-indistinguishability operator on $X$.

Theorem 2.6. Representation Theorem.[13] Let $R$ be a fuzzy relation on $X . R$ is a T-indistinguishability operator on $X$ if and only if there exists a family $\left(\mu_{i}\right)_{i \in I}$ of fuzzy subsets of $X$ such that $R=\inf _{i \in I} E_{\mu_{i}}$. 
Definition 2.7. [9] Let $\tau$ be a family of fuzzy subsets of $X$. $\tau$ is a fuzzy topology on $X$ if and only if for any family $\left(\mu_{i}\right)_{i \in I}$ of elements of $\tau, \mu, \nu \in \tau$

- $\bigvee_{i \in i} \mu_{i} \in \tau$

- $\mu \wedge \nu \in \tau$

- The constant fuzzy subsets of $X$ belong to $\tau$.

Definition 2.8. Let $X$ be a set. An operator $c:[0,1]^{X} \rightarrow[0,1]^{X}$ is a closure operator if and only if for all fuzzy subsets $\mu, \nu$ of $X$

- $\mu \subseteq c(\mu)$

- If $\mu \leq \nu$, then $c(\mu) \leq c(\nu)$

- $c(c(\mu))=c(\mu)$.

If moreover

- $c(\mu \vee \nu)=c(\mu) \vee c(\nu)$,

then $c$ is a topological closure operator.

\section{The Set of Extensional Fuzzy subsets with respect to an Indistinguishability Operator}

Definition 3.1. Let $E$ be a $T$-indistinguishability operator on a set $X$. A fuzzy subset $\mu$ of $X$ is extensional with respect to $E$ (or simply extensional) if and only if for all $x, y \in X$

$$
T(E(x, y), \mu(y)) \leq \mu(x) .
$$

$H_{E}$ will denote the set of extensional fuzzy subsets of $X$ with respect to $E$.

The previous definition fuzzifies the predicate

If $x$ and $y$ are equivalent and $y \in \mu$, then $x \in \mu$. 
If $E$ is a crisp equivalence relation on $X$, then a crisp subset $A$ of $X$ is extensional if and only if it is the union (and intersections if we want to obtain the empty set) of the equivalence classes of the elements of $A . H_{E}$ restricted to crisp subsets is in this case the set $\{0,1\}^{X / E}$ of subsets of the quotient set $X / E$. In other words, the set of the crisp extensional subsets of a crisp equivalence relation is generated by its equivalence classes. In a similar way we will prove in Proposition 3.8 that the extensional fuzzy subsets of an indistinguishability operator are generated by its fuzzy equivalence classes.

Proposition 3.2. Let $E$ be a T-indistinguishability operator on $X, \mu$ a fuzzy subset of $X$ and $E_{\mu}$ the T-indistinguishability operator generated by $\mu$ as in Proposition 2.4. $\mu \in H_{E}$ if and only if $E_{\mu} \geq E$.

Proof. $E_{\mu}(x, y)=E_{T}(\mu(x), \mu(y)) \geq E(x, y)$ if and only if $T(E(x, y), \mu(x)) \leq$ $\mu(y)$ and $T(E(x, y), \mu(y)) \leq \mu(x)$.

Definition 3.3. [14] Let $x \in X$ and $E$ a T-indistinguishability operator on $X$. The fuzzy equivalence class or column $\mu_{x}$ is the fuzzy subset of $X$ defined for all $y \in X$ by $\mu_{x}(y)=E(x, y)$.

N.B. If $E$ is a crisp equivalence relation, then $\left(\mu_{x}\right)_{x \in X}$ is the family of its equivalence classes.

Lemma 3.4. Given a T-indistinguishability operator $E$ on a set $X$ and an element $x \in X$, the column $\mu_{x}$ of $x$ is extensional.

Proof. Given $y, z \in X$

$$
E_{\mu_{x}}(y, z)=E_{T}(E(x, y), E(x, z)) \geq E(y, z) .
$$

The extensional fuzzy subsets with respect to a $T$-indistinguishability operator $E$ satisfy the following properties.

Proposition 3.5. [7] Let $E$ be a T-indistinguishability operator on a set $X$. The following properties are satisfied for all $\mu \in H_{E},\left(\mu_{i}\right)_{i \in I}$ a family of extensional fuzzy subsets and $\alpha \in[0,1]$.

1. $\bigvee_{i \in I} \mu_{i} \in H_{E}$.

2. $\bigwedge_{i \in I} \mu_{i} \in H_{E}$. 
3. $T(\alpha, \mu) \in H_{E}$.

4. $\vec{T}(\mu \mid \alpha) \in H_{E}$.

5. $\vec{T}(\alpha \mid \mu) \in H_{E}$.

Lemma 3.6. Let $E$ be a T-indistinguishability operator on a set $X . H_{E}$ is a generating family of $E$ in the sense of the Representation Theorem 2.6.

Proof. It is a straightforward consequence of Theorem 2.6.

There is a nice and important result in [7] that establishes a bijection between $T$-indistinguishability operators and subsets $H$ of $[0,1]^{X}$ satisfying the properties of Proposition 3.5.

Proposition 3.7. There is a bijection between T-indistinguishability operators and subsets $H$ of $[0,1]^{X}$ satisfying the properties of Proposition 3.5. If $E_{H}$ is the T-indistinguishability operator generated by the fuzzy subsets of by $H$, then $E_{H_{E}}=E$ and $H_{E_{H}}=H$.

Reinterpreting the proof of this result, we obtain the important fact that the extensional fuzzy subsets of $E$ can be obtained (are combinations) of the fuzzy equivalence classes of $E$, which generalizes the fact that the observable crisp subsets of a crisp equivalence relation are unions of its equivalence classes.

Also this result will be extended to characterizing the sets of extensional mappings $f: X \rightarrow Y$ in Section 4 .

Proposition 3.8. Let $F C$ be the set of fuzzy equivalence classes or columns of a T-indistinguishability operator $E$ on a set $X$ (i.e., $F C=\left\{\mu_{y} \in[0,1]^{X} \mid \mu_{y}(x)=\right.$ $E(x, y) \forall x, y \in X\})$. Then $H_{E}$ is the smallest subset of $[0,1]^{X}$ closed by the operations of Proposition 3.5 and containing FC.

Proof. Given $\mu \in H_{E}$ and $y \in X$, let us define the fuzzy subset $\mu^{y}$ by $\mu^{y}(x)=T\left(\mu_{y}(x), \mu(y)\right)$ for all $x \in X$.

$$
\begin{aligned}
\mu^{y}(x) & =T\left(\inf _{\nu \in H_{E}} E_{\nu}(x, y), \mu(y)\right) \\
& =\inf _{\nu \in H_{E}} T(\min (\vec{T}(\nu(x) \mid \nu(y)), \vec{T}(\nu(y) \mid \nu(x))), \mu(y)) .
\end{aligned}
$$

Since $\mu(y)$ and $\nu(y)$ are constants, applying properties $2,3,4,5$ of Proposition 3.5 we have that $\mu^{y} \in H_{E}$. 
Since $\mu$ is extensional, $\mu^{y}(x)=T(E(x, y), \mu(y)) \leq \mu(x)$. Adding that $\mu^{y}(y)=\mu(y)$ we obtain $\mu=\sup _{y \in X} \mu^{y}$ which is an element of $H_{E}$ tanks to property 3.5.1.

Finally, pointing out that for every $y \in X$ and $\mu \in H_{E} \mu(y)$ is a constant, we obtain $\mu$ from the columns of $E$ applying the operations of Proposition 3.5 .

N.B. The obtained expression for $\mu: \mu(x)=\sup _{y \in X} T\left(\mu_{y}(x), \mu(y)\right)$ fuzzifies the predicate

$x \in \mu$ if and only if there exists an equivalence class [y] of $E$ such that $x \in[y]$ and $[y] \subseteq \mu$.

In other words, in the crisp case $\mu$ is the union of equivalence classes of $E$.

Proposition 3.9. [8] $H_{E}$ is a fuzzy topology.

In the study of the extensionality of fuzzy subsets and, as we will see, in the study of general extensional mappings, $\left([0,1], E_{T}\right)$ plays a central role. It therefore deserves a special attention and the next results characterize the sets $H_{E_{T}}$ for specific t-norms.

Proposition 3.10. Let $T$ be the minimum t-norm. Then $\mu \in H_{E_{T}}$ if and only if for all $x, y \in[0,1]$

$$
\min (x, y, \mu(y)) \leq \mu(x)
$$

Proof.

$\mu \in H_{E_{T}}$ if and only if $\min \left(E_{T}(x, y), \mu(y)\right) \leq \mu(x)$ for all $x, y \in[0,1]$.

- If $x=y$, then $\min \left(E_{T}(x, y), \mu(y)\right)=\min (1, \mu(y))=\mu(x)$.

- If $x \neq y$, then $\min \left(E_{T}(x, y), \mu(y)\right)=\min (x, y, \mu(y))$.

In [11] the following alternative characterization is provided.

Proposition 3.11. Let $T$ be the minimum t-norm. Then $\mu \in H_{E_{T}}$ if and only if for all $x \in[0,1] \mu(x)>x$ for all $x \leq u=\inf _{z \in[0,1]}\{\mu(z)<z\}$ and $\mu(x)=u$ for all $x>u$. 
Definition 3.12. Let $c>0$. A real function $f: \mathbb{R} \rightarrow \mathbb{R}$ is c-Lipschitz if and only if for all $x, y \in \mathbb{R}$

$$
|f(x)-f(y)| \leq c|x-y| .
$$

Proposition 3.13. [11] Let $t$ be an additive generator of a continuous Archimedean $t$-norm $T$. Then $\mu \in H_{E_{T}}$ if and only if the function $t \circ \mu \circ t^{-1}$ : $[0, t(0)] \rightarrow[0, t(0)]$ is 1-Lipschitz.

In particular,

Proposition 3.14. Let $T$ be the Eukasiewicz t-norm. Then $\mu \in H_{E_{T}}$ if and only if $\mu$ is 1-Lipschitz.

Proof.

$$
\mu \in H_{E_{T}} \text { if and only if } 1-|\mu(x)-\mu(y)| \geq 1-|x-y|
$$

which is equivalent to

$$
|\mu(x)-\mu(y)| \leq|x-y|
$$

Proposition 3.15. Let $T$ be the Product t-norm. Then $\mu \in H_{E_{T}}$ if and only if $\frac{\mu(x)}{x}$ is a non increasing and $x \cdot \mu(x)$ a non decreasing function for all $x>0$.

Proof. Though the result can be obtained as a corollary of Proposition 3.13, a direct proof will be given.

$\mu \in H_{E_{T}}$ if and only if $\min \left(\frac{x}{y}, \frac{y}{x}\right) \cdot \mu(y) \leq \mu(x)$.

If $x \leq y$, the last inequality is equivalent to

$$
\frac{x}{y} \cdot \mu(y) \leq \mu(x)
$$

and

$$
\frac{x}{y} \cdot \mu(x) \leq \mu(y)
$$

Corollary 3.16. If $T$ is the product $t$-norm and $\mu \in[0,1]^{[0,1]}$ is a differentiable function, then $\mu \in H_{E_{T}}$ if and only if $\frac{\mu(x)}{x} \geq\left|\mu^{\prime}(x)\right|$. 
Proof.

- $\frac{\mu(x)}{x}$ is a non-increasing function if and only if its derivative is less than or equal to zero:

$$
\frac{\mu^{\prime}(x) \cdot x-\mu(x)}{x^{2}} \leq 0
$$

or

$$
\frac{\mu(x)}{x} \geq \mu^{\prime}(x)
$$

- $x \cdot \mu(x)$ is a non-decreasing function if and only if its derivative is greater than or equal to zero:

$$
\mu(x)+x \mu^{\prime}(x) \geq 0
$$

or

$$
\frac{\mu(x)}{x} \geq-\mu^{\prime}(x) .
$$

Therefore,

$$
\frac{\mu(x))}{x} \geq\left|\mu^{\prime}(x)\right|
$$

Example 3.17. The fuzzy subsets $\mu_{\alpha}$ of $[0,1]$ defined by $\mu_{\alpha}(x)=x^{\alpha}$ for all $x \in[0,1](0 \leq \alpha \leq 1)$ are extensional sets of $E_{T}$ with $T$ the Product $t$-norm.

Proposition 3.18. $\mu \in H_{E_{T}}$ if and only if for all fuzzy subsets $\nu$ of $[0,1]$, $E_{\nu} \leq E_{\mu \circ \nu}$.

Proposition 3.19. Let $t$ be an additive generator of a continuous strict Archimedean $t$-norm $T$ with $t(0) \leq 1 . t \in H_{E_{T}}$ if and only if $t$ is 1-Lipschitz.

Proof. It is a consequence of Proposition 3.13.

\section{Extensionality of Mappings}

In this section, the extensionality of a mapping $f: X \rightarrow Y$ will be studied. The main results are:

- The equivalence between extensionality and fuzzy continuity will be proved. 
- A characterization of the set of extensional mappings for given $X$ and $Y$ will be provided.

Definition 4.1. Let $E$ and $F$ be T-indistinguishability operators on $X$ and $Y$ respectively. $f: X \rightarrow Y$ is extensional with respect to $E$ and $F((E, F)$ extensional) if and only if for $x, x^{\prime} \in X, E\left(x, x^{\prime}\right) \leq F\left(f(x), f\left(x^{\prime}\right)\right)$.

Let us recall the operator $\phi_{E}$ that provides the best upper approximations of a fuzzy subset by an extensional one.

Definition 4.2. [8] Let $E$ be a T-indistinguishability operator on a set $X$. The map $\phi_{E}:[0,1]^{X} \rightarrow[0,1]^{X}$ is defined by

$$
\phi_{E}(\mu)(x)=\sup _{y \in X} T(E(x, y), \mu(y)), \forall x \in X .
$$

$\phi_{E}$ is $(\bar{E}, \bar{E})$-extensional where $\bar{E}$ is the $T$-indistinguishability operator $\bar{E}$ on $[0,1]^{X}$ defined for all fuzzy subsets $\mu, \nu$ of $X$ by $\bar{E}(\mu, \nu)=\inf _{x \in X} E_{T}(\mu(x), \nu(x))$.

Proposition 4.3. [8] Let E be a T-indistinguishability operator on $X$. Then $\phi_{E}$ is a fuzzy closure operator.

Proposition 4.4. [8]

- $\operatorname{Im} \phi_{E}=H_{E}$

- $\mu \in H_{E}$ if and only if $\phi_{E}(\mu)=\mu$

- $\mu \in[0,1]^{X}, \phi_{E}(\mu)=\bigwedge_{\mu^{\prime} \in H_{E}}\left\{\mu \leq \mu^{\prime}\right\}$.

So, $\phi_{E}(\mu)$ is the most specific extensional fuzzy subset that contains $\mu$ (i.e. $\mu \leq \phi_{E}(\mu)$ ), and is the optimal upper bound of $\mu$ in $H_{E}$.

Definition 4.5. Let $f: X \rightarrow Y$ be a mapping and $\nu$ a fuzzy subset of $Y$. $f^{-1}(\nu)$ is the fuzzy subset of $X$ defined by

$$
f^{-1}(\nu)(x)=\nu(f(x))
$$

for all $x \in X$.

Definition 4.6. Let $\sigma$ be a fuzzy topology on $X$ and $\tau$ a fuzzy topology on $Y$. A mapping $f: X \rightarrow Y$ is fuzzy-continuous with respect to $\sigma$ and $\tau$, or simply $\varphi$-continuous, if and only if for every $\nu \in \tau, f^{-1}(\nu) \in \sigma$. 
Proposition 4.7. Let $E$ and $F$ be $T$-indistinguishability operators on $X$ and $Y$ respectively. $f: X \rightarrow Y$ is $\varphi$-continuous with respect to $H_{E}$ and $H_{F}$ if and only if $f$ is $(E, F)$-extensional.

Proof.

$\Rightarrow$ Let $\nu \in H_{F}$. Since $f$ is $\varphi$-continuous, $f^{-1}(\nu) \in H_{E}$, i.e., for all $x, x^{\prime} \in X$

$$
T\left(f^{-1}(\nu)(x), E\left(x, x^{\prime}\right)\right) \leq f^{-1}(\nu)\left(x^{\prime}\right)
$$

or

$$
T\left(\nu(f(x)), E\left(x, x^{\prime}\right)\right) \leq \nu\left(f\left(x^{\prime}\right)\right)
$$

which means

$$
E\left(x, x^{\prime}\right) \leq E_{\nu}\left(f(x), f\left(x^{\prime}\right)\right) .
$$

This last inequality is valid for all $\nu \in H_{F}$ and since $F=\inf _{\nu \in H_{F}} E_{\nu}$,

$$
E\left(x, x^{\prime}\right) \leq F\left(f(x), f\left(x^{\prime}\right)\right)
$$

and $f$ is extensional.

$\Leftarrow)$ Let $\nu \in H_{F}$. Then $\nu=\phi_{F}(\nu)$ and we must prove that, assuming $f$ extensional, $f^{-1}\left(\phi_{F}(\nu)\right) \in H_{E}$.

$$
\begin{aligned}
T & \left(f^{-1}\left(\phi_{F}(\nu)\right)(x), E\left(x, x^{\prime}\right)\right) \\
& =T\left(\phi_{F}(\nu)(f(x)), E\left(x, x^{\prime}\right)\right) \\
& =\sup _{y \in Y} T\left(\nu(y), F(y, f(x)), E\left(x, x^{\prime}\right)\right) \\
& \leq \sup _{y \in Y} T\left(\nu(y), F(y, f(x)), F\left(f(x), f\left(x^{\prime}\right)\right)\right) \\
& \leq \sup _{y \in Y} T\left(\nu(y), F\left(y, f\left(x^{\prime}\right)\right)\right) \\
& =\phi_{F}\left(\nu\left(f\left(x^{\prime}\right)\right)=f^{-1}\left(\phi_{F}(\nu)\right)\left(x^{\prime}\right) .\right.
\end{aligned}
$$

Corollary 4.8. A fuzzy subset $\mu$ of $X$ is extensional with respect to a $T$ indistinguishability operator $E$ on $X$ if and only if $\mu$ is $\varphi$-continuous with respect to $H_{E}$ and $H_{E_{T}}$. 
We now will try to characterize the set of extensional mappings $f: X \rightarrow$ $Y$.

Proposition 4.9. Let $E$ and $F$ be $T$-indistinguishability operators on $X$ and $Y$ respectively. $f: X \rightarrow Y$ is extensional if and only if $\mu \circ f$ is an extensional fuzzy subset of $X$ for all extensional fuzzy subsets $\mu$ of $Y$.

Proof.

$\Rightarrow)$ If $f$ is $(E, F)$-extensional and $\mu \in H_{F}$, then

$$
E\left(x, x^{\prime}\right) \leq F\left(f(x), f\left(x^{\prime}\right)\right) \leq E_{T}\left(\mu(f(x)), \mu\left(f\left(x^{\prime}\right)\right)\right) .
$$

$\Leftarrow)$

$$
E\left(x, x^{\prime}\right) \leq E_{T}\left(\mu(f(x)), \mu\left(f\left(x^{\prime}\right)\right)\right) \text { for all } \mu \in H_{F}
$$

and therefore

$$
E\left(x, x^{\prime}\right) \leq \inf _{\mu \in H_{F}} E_{T}\left(\mu(f(x)), \mu\left(f\left(x^{\prime}\right)\right)\right)=F\left(f(x), f\left(x^{\prime}\right)\right) .
$$

The next simple example shows that in general we cannot recover the original $T$-indistinguishability operator $E$ from the set of extensional mappings $f: X \rightarrow Y$, but a greater one

Example 4.10. Let $E$ be a T-indistinguishability operator on $X$ and $Y=$ $\{y\}$ a set of cardinality 1 . The only $T$-indistinguishability operator $F$ on $Y$ is defined by $F(y, y)=1$ and all fuzzy subsets of $Y$ (constant mappings) are extensional with respect to $Y$. The only mapping $f: X \rightarrow Y(f(x)=y$ for all $x \in X)$ is extensional and $\mu \circ f$ is a constant fuzzy subset of $X$ for all fuzzy subsets $\mu$ of $Y$. So the set $\left\{\mu_{i} \circ f \mid \mu_{i}\right.$ a fuzzy subset of $\left.Y\right\}$ generates the universal T-indistinguishability operator $E^{\prime}$ on $X\left(E^{\prime}\left(x, x^{\prime}\right)=1\right.$ for all $\left.x, x^{\prime} \in X\right)$ which is greater than or equal to the original $E$.

In general, the result is the following one.

Proposition 4.11. Let $E$ and $F$ be $T$-indistinguishability operators on $X$ and $Y$ respectively. Consider the set $A$ of extensional mappings $f:(X, E) \rightarrow$ $(Y, F)$. the set $M=\left\{\mu_{i} \circ f_{j} \mid \mu_{i} \in H_{F}, f_{j} \in A\right\}$ generates the greatest $T$-indistinguishability $E^{\prime}$ on $X$ containing $E$ and with $A$ the set of extensional mappings $f:\left(X, E^{\prime}\right) \rightarrow(Y, F)$. 
Proof. The fuzzy subsets $\mu_{i} \circ f_{j}$ are extensional with respect to $E$ and hence $M$ generates a $T$-indistinguishability operator $E^{\prime}$ on $X$ greater than or equal to $E$ and with $A$ the set of $\left(E^{\prime}, F\right)$-extensional mappings.

Proposition 4.12. Let $E$ and $F$ be $T$-indistinguishability operators on $X$ and $Y$ respectively, $A$ the set of $(E, F)$-extensional mappings $f: X \rightarrow Y$. The following properties are satisfied for all $\mu \in H_{F},\left(\mu_{i}\right)_{i \in I}$ a family with $\mu_{i} \in H_{F}$ for all $i \in I, f \in A,\left(f_{j}\right)_{j \in J}$ a family with $f_{j}: X \rightarrow Y, f_{j} \in A$ for all $j \in J$ and $\alpha \in[0,1]$.

1. $\bigvee_{i \in I, j \in J} \mu_{i} \circ f_{j} \in H_{E}$.

2. $\bigwedge_{i \in I, j \in J} \mu_{i} \circ f_{j} \in H_{E}$.

3. $T(\alpha, \mu \circ f) \in H_{E}$.

4. $\vec{T}(\mu \circ f \mid \alpha) \in H_{E}$.

5. $\vec{T}(\alpha \mid \mu \circ f) \in H_{E}$.

Proof. It is a consequence of 3.5 .

Proposition 4.13. Let $X$ and $Y$ be sets, $F$ a T-indistinguishability operator on $Y$ and $A$ a set of mappings $f: X \rightarrow Y$ satisfying the properties of Proposition 4.12. Then there exists a T-indistinguishability operator $E^{\prime}$ on $X$ such that

- $A$ is the set of extensional mappings $f:\left(X, E^{\prime}\right) \rightarrow(Y, F)$

- $E^{\prime}$ is the greatest T-indistinguishability operator satisfying the previous property.

Proof. $E^{\prime}$ is the $T$-indistinguishability operator on $X$ generated by $M=$ $\left\{\mu_{i} \circ f_{j} \mid \mu_{i} \in H_{F}, f_{j} \in A\right\}$

\section{Type-2 Fuzzy Sets and Extensionality}

For a number of reasons in many situations it is not clear how to assign an exact membership value $\mu(x)$ of the unit interval to an object $x$ of a universe $X$. This kind of uncertainty cannot be incorporated to $\mu$ directly but in these cases $\mu$ must be fuzzified by allowing that the membership value be a fuzzy 
subset on the unit interval. This is the main reason to consider more general fuzzy subsets called type-2 fuzzy subsets in the literature. (In opposition, classical fuzzy subsets will be called type-1 fuzzy subsets). In this section this interpretation of type-2 fuzzy subsets as fuzzification of type- 1 fuzzy subsets is provided by considering extensionality and a special kind of fuzzy mappings. Readers interested in type-2 fuzzy subsets and their applications are referred to [10] and to the bibliography included in it.

Fuzzy mappings generalize the concept of mapping between two sets $X$ and $Y$. The sets are supposed to be endowed with $T$-indistinguishability operators and compatibility of the fuzzy mappings with them is imposed. Interesting properties of fuzzy mappings can be found in [5] [6].

Definition 5.1. Let $E_{X}$ and $E_{Y}$ be T-indistinguishability operators on two sets $X$ and $Y$ respectively. $R: X \times Y \rightarrow[0,1]$ is a fuzzy mapping from $X$ onto $Y$ if and only if for all $x, x^{\prime} \in X$ and for all $y, y^{\prime} \in Y$

- $T\left(R(x, y), E_{X}\left(x, x^{\prime}\right), E_{Y}\left(y, y^{\prime}\right)\right) \leq R\left(x^{\prime}, y^{\prime}\right)$ (Extensionality)

- $T\left(R(x, y), R\left(x, y^{\prime}\right)\right) \leq E_{Y}\left(y, y^{\prime}\right)$ (Functionality).

$R$ is perfect if and only if

- For all $x \in X$ there exists a unique $y \in Y$ such that $R(x, y)=1$.

$R(x, y)$ is the degree to which $y$ is the image of $x$.

Proposition 5.2. [5] There exists a bijection between perfect fuzzy mappings and extensional mappings.

- From an extensional mapping $f: X \rightarrow Y$, we obtain $R_{f}(x, y)=$ $F(f(x), y)$.

- Given the fuzzy mapping $R$, the corresponding crisp extensional mapping is $f_{R}(x)=y_{0}$, where $y_{0}$ is the only element of $Y$ with $R(x, y)=1$.

Now consider an extensional fuzzy subset $\mu$ of $X$ with respect to $E$. This is equivalent to the fact that $\mu: X \rightarrow[0,1]$ is $\left(E, E_{T}\right)$-extensional. So $\mu$ generates the fuzzy mapping $R_{\mu}: X \times[0,1] \rightarrow[0,1]$ defined for all $x \in X$ and $\alpha \in[0,1]$ by

$$
R_{\mu}(x, \alpha)=E_{T}(\mu(x), \alpha)
$$


and therefore the (type-1) fuzzy subset $\mu$ generates a type-2 fuzzy subset on $X$ :

$$
\begin{aligned}
X & \rightarrow[0,1]^{[0,1]} \\
x & \rightarrow R_{\mu}(x, \cdot):[0,1] \rightarrow[0,1] .
\end{aligned}
$$

In particular, if $E$ is the crisp equality on $X(E(x, y)=1$ if $x=y$ and $E(x, y)=0$ otherwise), then all fuzzy subsets are extensional and hence every fuzzy subset of $X$ generates a type-2 fuzzy subset of $X$.

Example 5.3. If $T$ is the Eukasiewicz t-norm and $\mu$ a fuzzy subset of $X$, then the type-2 fuzzy subset $R_{\mu}$ of $X$ generated by $\mu$ is

$$
R_{\mu}(x, \alpha)=1-|\mu(x)-\alpha| .
$$

In other words, for a fixed $x \in X, R_{\mu}(x, \cdot)$ is a symmetric triangular fuzzy subset (of $[0,1])$ centered at $\mu(x)$.

Of course the $T$-indistinguishability operator $E_{T}$ on $[0,1]$ can be replaced by another such operator $F$. In this way a fuzzy subset $\mu$ of $X$ can generate multiple type-2 fuzzy subsets according to the diverse granularity generated by $F$ on $[0,1]$.

Example 5.4. Continuing with Example 5.3, we can consider powers of $E_{T}$ $\left(E_{T}^{k}(x, y)=\max (0,1-k|x-y|, k \geq 0)\right.$. Then $R_{\mu}(x, \alpha)$ is the triangular fuzzy subset of $[0,1]$ with core $\mu(x)$ and slopes $\pm k$.

Reciprocally, let us see when a (type-1) fuzzy subset can be obtained from a given type- 2 one. This will be the case when it determines a perfect fuzzy mapping from $X$ to $[0,1]$. Since in $X$ we consider $E$ to be the crisp equality, we are in the case called classic in [12]. The elements of the domain are considered crisp and completely distinguishable between them and there is only vagueness or uncertainty in their images.

Classical fuzzy mappings appear in many contexts, since many times we know exactly the elements of our domain and are completely distinguishable. Then we only are uncertain about which exact image each elements of the domain has. This is the case for type-2 fuzzy subsets.

Definition 5.5. A classical fuzzy mapping $R$ from $X$ onto $Y$ is a fuzzy mapping on $X \times Y$ with $E$ the classical crisp equality on $X$ and $F$ a $T$ indistinguishability operator on $Y$. 
Definition 5.6. A fuzzy subset $\mu$ on $X$ is a fuzzy point of $X$ with respect to a given $T$-indistinguishability operator $E$ on $X$ if and only if

- $\mu \in H_{E}$.

- $T\left(\mu(x), \mu\left(x^{\prime}\right)\right) \leq E\left(x, x^{\prime}\right)$ for all $x, x^{\prime} \in X$.

Proposition 5.7. [3] The normal fuzzy points of $E$ are exactly its columns.

Proposition 5.8. A fuzzy subset $R$ of $X \times Y$ is a classical fuzzy mapping on $X \times Y$ with $F$ a T-indistinguishability operator on $Y$ if and only if $R(x, \cdot)$ is a fuzzy point of $Y$ with respect to $F$ for all $x \in X$.

Proof. Extensionality of $R$ is

$$
T\left(F\left(y, y^{\prime}\right), R(x, y)\right) \leq R\left(x, y^{\prime}\right)
$$

for all $x \in X, y, y^{\prime} \in Y$, which is equivalent to say that $R(x, \cdot)$ is extensional with respect to $F$. The condition of functionality

$$
T\left(R(x, y), R\left(x, y^{\prime}\right) \leq F\left(y, y^{\prime}\right)\right.
$$

also expresses that $R(x, \cdot)$ is a fuzzy point for all $x \in X$.

From a classical fuzzy mapping on $X \times Y$ we have a function $f: X \rightarrow$ $\mathcal{F}(Y)$ where $\mathcal{F}(Y)$ is the set of fuzzy subsets of $Y$ by simply defining $f(x)=$ $R(x, \cdot)$.

Reciprocally,

Proposition 5.9. Let $f: X \rightarrow \mathcal{F}(Y)$ be a function from $X$ to the set $\mathcal{F}(Y)$ of fuzzy subsets of $Y$ and $R$ the fuzzy subset of $X \times Y$ defined by $R(x, y)=F(f(x), y)$ for all $x, y \in X \times Y$. R is a classical fuzzy mapping on $X \times Y$ with $F$ a T-indistinguishability operator on $Y$ if and only if for all $x \in X R(x, \cdot)$ is a fuzzy point of $Y$.

$R$ is perfect if and only if the fuzzy points are normal; i.e., columns of $F$.

Specifying these results to the case $Y=[0,1]$, we obtain the characterization of type-2 fuzzy subsets from which a type-1 fuzzy subset can be derived.

Proposition 5.10. A type-2 fuzzy subset $R$ of $X$ is a fuzzy mapping on $X \times[0,1]$ with $F$ a T-indistinguishability operator on $[0,1]$ if and only if $R(x, \cdot)$ is a normal fuzzy point of $[0,1]$ with respect to $F$ for all $x \in X$. 
Example 5.11. Let $F$ be a T-indistinguishability operator on $[0,1]$. Its columns are normal fuzzy points and so the type-2 fuzzy subset of $X$ assigning a column $\mu_{f(x)}$ of $F$ to any $x \in X$ generates a perfect fuzzy mapping from $X$ to $[0,1]$ and the associated type-1 fuzzy subset $\mu$ is defined by $\mu(x)=f(x)$.

\section{Concluding Remarks}

In this paper different aspects of extensionality of mappings and fuzzy subsets have been dealt with. Extensionality gives coherence between crisp or fuzzy objects with respect to fuzzy equivalence relations and it is why it is also called compatibility [1]. Extensionality can also be defined on products of universes $X=X_{1} \times X_{2} \times \ldots \times X_{n}$ with corresponding $T$-indistinguishability operators $E_{1}, E_{2}, \ldots, E_{n}$. But in this case, the relation $E=T\left(E_{1}, E_{2}, \ldots, E_{n}\right)$ is a

$T$-indistinguishability operator and extensionality with respect to $E_{1}, E_{2}, \ldots, E_{n}$ is equivalent to extensionality with respect to $E$.

Let us finish this paper with a look at Zadeh's Extension Principle [15] form an extensional point of view.

The extension Principle allows us to extend a mapping $f: X \rightarrow Y$ to a mapping $f:[0,1]^{X} \rightarrow[0,1]^{Y}$ between their sets of fuzzy subsets.

Definition 6.1. [15] Let $f: X \rightarrow Y$ be a mapping, The mapping $f$ : $[0,1]^{X} \rightarrow[0,1]^{Y}$ is defined for all $\mu \in[0,1]^{X}$ by

$$
f(\mu)(y)=\sup _{x \in X, f(x)=y} \mu(x) .
$$

If there are defined $T$-indistinguishability operators $E$ and $F$ on $X$ and $Y$ respectively, we would like $f$ to be compatible with them. A coherent condition is that the images of the fuzzy equivalence classes of $E$ be included in the fuzzy equivalence classes of $F$. This can be assured when $f$ is extensional.

Proposition 6.2. Let $E$ and $F$ be two T-indistinguishability operators on $X$ and $Y$ respectively, $f: X \rightarrow Y, a \in X$ and $\mu_{a}$ its corresponding equivalence class (i.e.: $\left.\mu_{a}(x)=E(a, x)\right)$. If $f$ is $(E, F)$-extensional, then $f\left(\mu_{a}\right) \leq \mu_{f(a)}$.

Proof.

$$
\begin{aligned}
f\left(\mu_{a}\right)(y) & =\sup _{x \in X, f(x)=y} \mu_{a}(x)=\sup _{x \in X, f(x)=y} E(a, x) \\
& \leq \sup _{x \in X, f(x)=y} F(f(a), f(x))=F(f(a), y)=\mu_{f(a)}(y) .
\end{aligned}
$$


In order to obtain a mapping between fuzzy subsets compatible with $E$ and $F$, we propose the following definition.

Definition 6.3. Let $E$ and $F$ be two T-indistinguishability operators on $X$ and $Y$ respectively, $f: X \rightarrow Y$. The mapping $\bar{f}:[0,1]^{X} \rightarrow[0,1]^{Y}$ is defined for all $\mu \in[0,1]^{X}$ by

$$
\bar{f}(\mu)=\phi_{F}\left(f\left(\phi_{E}(\mu)\right) .\right.
$$

The study of the mapping $\bar{f}$ and its relation with extensionality will be developed by the authors in a forthcoming work.

\section{References}

[1] R. Bělohlávek Fuzzy Relational Systems. Foundations and Principles. Springer, 2002.

[2] D. Boixader, J. Jacas Extensionality based approximate reasoning International Journal of Approximate Reasoning 19 (1998) 221-230.

[3] D. Boixader, J. Recasens. A map characterizing the fuzzy points and columns of a T-indistinguishability operator. Int. J. Uncertainty, Fuzziness and Knowledge-based Systems 16 (2008) 129-145.

[4] D. Boixader, J. Jacas, J. Recasens Upper and lower approximation of fuzzy sets. Int. J. of General Systems 29 (2000) 555-568.

[5] M. Demirci Fuzzy functions and their fundamental properties. Fuzzy Sets and Systems 106 (1999) 239-246.

[6] M. Demirci, J. Recasens Fuzzy groups, fuzzy functions and fuzzy equivalence relations. Fuzzy Sets and Systems 144 (2004) 441-458.

[7] J.L. Castro, F. Klawonn Similarity in Fuzzy Reasoning. Mathware \& Soft Computing 2 (1996) 197-228.

[8] J. Jacas, J. Recasens Fuzzy T-transitive relations: eigenvectors and generators. Fuzzy Sets and Systems 72 (1995) 147-154. 
[9] R. Lowen Fuzzy topological spaces and fuzzy compactness. Journal of Mathematical Analysis and Applications 56 (1976) 623-633.

[10] J.M. Mendel, R.I. John Type-2 Fuzzy Sets Made Simple. IEEE Transactions on Fuzzy Systems 10 (2002) 117-127.

[11] R. Mesiar, V. Novák Operations fitting triangular-norm-based biresiduation Fuzzy Sets and Systems 104 (1999) 77-84.

[12] J: Recasens Indistinguishability Operators. Modelling Fuzzy Equalities and Fuzzy Equivalence Relations. Studies in Fuzziness and Soft Computing. Springer, 2011.

[13] L. Valverde On the Structure of F-indistinguishability Operators. Fuzzy Sets and Systems 17 (1985) 313-328.

[14] L.A. Zadeh Similarity relations and fuzzy orderings. Inf. Sciences 3 (1971) 177-200.

[15] 5. L.A. Zadeh The concept of a linguistic variable and its applications in aproximate reasoning. Inf. Sciences 8 (1975) 199-251, 301-357, 9 (1975) 43-80. 